\title{
Evaluación de secuencias de aprendizaje de matemáticas usando la herramienta de los Criterios de Idoneidad Didáctica
}

\author{
Using the Didactical Suitability Criteria tool when assessing \\ mathematics learning sequences
}

\author{
Orlando García Marimón* \\ ORCID iD 0000-0002-0334-6133 \\ Javier Diez-Palomar** \\ ORCID iD 0000-0003-4447-1595 \\ Luisa Morales Maure ${ }^{* * *}$ \\ ORCID iD 0000-0003-3905-9002 \\ Rosa Elena Durán González ${ }^{* * * *}$ \\ ORCID iD 0000-0001-8121-5019
}

\begin{abstract}
Resumen
En este artículo se utiliza la herramienta de análisis didáctico denominada Criterios de Idoneidad Didáctica (CID) para valorar las secuencias didácticas elaboradas por maestros que han cursado un Diplomado en la Universidad de Panamá. Dicha herramienta se ha desarrollado en el marco del Enfoque Ontosemiótico (EOS), revelándose útil para este tipo de acciones, tal como lo han validado las investigaciones previas. En esta investigación se propone un uso sistemático de los CID, aplicando una escala ordinal (tipo Likert) de cuatro posiciones, para cuantificar cada uno de los criterios y, así, evaluar las secuencias didácticas. Los investigadores (uno de los cuales es el formador de los docentes) discuten las secuencias didácticas elaboradas por los docentes, a partir de las grabaciones de aula, donde el análisis les permite sacar conclusiones sobre las decisiones didácticas que realizan los maestros. El uso de los CID revela fortalezas y debilidades en las secuencias didácticas de los maestros. El análisis de la idoneidad didáctica de las secuencias didácticas presentadas por los y las maestras sugiere que el Diplomado ha servido para mejorar su competencia de análisis didáctico, aunque también se han encontrado evidencias que sugieren que la experiencia previa docente aporta elementos importantes que explican el mayor uso de algunos criterios para orientar su práctica.
\end{abstract}

Palabras clave: Criterios de Idoneidad Didáctica. Formación del Profesorado de Matemáticas. Evaluación.

\footnotetext{
* Doctorando en Didáctica de las Matemáticas y las Ciencias Experimentales de la Universitat de Barcelona (UB). Profesor del Departamento de Matemática de la Universidad de Panamá (UP), Panamá, Panamá. E-mail: orlando.egarcia@up.ac.pa.

** Doctor en Educación Matemática por la Universitat de Barcelona (UB). Profesor de Didáctica de las Matemáticas del Departamento de Educación Lingüística y Literaria, y Didáctica de las Ciencias Experimentales y de la Matemática, de la Universidad de Barcelona (UB), Barcelona, España. E-mail: jdiezpalomar@ub.edu.

*** Doctora en Didáctica de las Matematicas y las Ciencias Experimentales por la Universitat de Barcelona (UB), España. Profesora del Departamento de Matemática de la Universidad de Panamá (UP), Panamá, Panamá. E-mail: luisa.morales@up.ac.pa.

**** Doctorado en Ciencias de la Educación por la Universidad Autónoma del Estado de Hidalgo (UAEH). Profesora Investigadora del Área Académica de Ciencias de la Educación, de la Línea de Estudios Sociales y Culturales en Educación, Currículum y Evaluación, Universidad Autónoma del Estado de Hidalgo (UAEH), Pachuca, Hidalgo, México. E-mail: rdurango@uaeh.edu.mx.
} 


\begin{abstract}
This article uses the didactic analysis tool called Didactic Suitability Criteria (DSC) to assess the didactic sequences developed by teachers who have completed a qualified course at the University of Panama. This tool has been developed within the framework of the OntoSemiotic Approach (OSA), proving useful for this type of action, as validated by previous research. In this research, a systematic use of DSCs is proposed by applying an ordinal scale (Likert type) with four positions to quantify each of the criteria and thus evaluate the didactic sequences. The researchers (one of whom is the teacher trainer) discussed the didactic sequences developed by the teachers, based on the classroom recordings, where the analysis allowed them to draw conclusions about the didactic decisions made by the teachers. The use of DSCs reveals strengths and weaknesses in the teachers' teaching sequences. The analysis of the didactic sequences' suitability presented by the teachers suggests that the qualified course has served to improve their competence in didactic analysis, although evidence has also been found suggesting that previous teaching experience also provides important elements that explain a greater suitability in some criteria considered in the analysis.
\end{abstract}

Keywords: Didactic Suitability Criteria. Mathematics Teacher Training. Assessment.

\title{
1 Introducción
}

El estudio, que se discute aquí, se enmarca en la línea de la formación de los maestros/as de matemáticas. La formación de los profesionales de la educación es una de las variables que explican las diferencias de resultados educativos que existen entre diferentes países (KUNTER et al., 2013). Los estudios clásicos, en esta línea de investigación, han mostrado que para enseñar matemáticas no es suficiente conocer la disciplina; además, los maestros/as de matemáticas también necesitan desarrollar lo que, actualmente, llamamos competencia didáctica y competencia de análisis didáctico.

Shulman (1986) fue el primero en hablar del concepto de pedagogical content knowledge (PCK), que después ha sido ampliamente estudiado en investigaciones posteriores (CARPENTER et al., 1988, HILL; BALL; SCHILLING, 2008; ÜNVER; ÖZGÜR; GÜZEL, 2020; YOUNG et al., 2019). Actualmente, existen diversas aproximaciones teóricas que estudian el conocimiento que deben adquirir los maestros de matemáticas, por ejemplo, el enfoque del Conocimiento Especializado del Profesor de Matemáticas (ROWLAND et al., 2009); el enfoque del Mirar Profesionalmente o Noticing (MASON, 2011; LLINARES, 2012); el enfoque de la Práctica Reflexiva (SCHÖN, 1983) entre otros.

En este artículo, nos centramos en la perspectiva del Modelo de Conocimientos y Competencias didáctico-Matemáticas del Profesor de Matemáticas (modelo CCDM) (BREDA; PINO-FAN; FONT, 2017; GODINO et al., 2017; PINO-FAN; FONT; BREDA, 2017).

En 2015, la UNESCO, en cooperación con la Universidad Nacional de Seúl, desarrolló un conjunto de indicadores globales de competitividad y aprendizaje permanente, organizados en base a cuatro pilares: saber, ser, hacer y convivir ( $\mathrm{OH}$ et al., 2015). En este estudio se 
agruparon los países en tres grupos, de acuerdo con su capacidad de competitividad y de aprendizaje: fuertes, intermedios y frágiles. En el caso de la República de Panamá, se sitúa en el grupo de los países considerados frágiles. Según este informe, la formación profesional del profesorado en Panamá es poco actual, descontextualizada, discontinua (los maestros solo se forman durante dos semanas cada año) y no es valorada para su promoción profesional. Estos datos sugieren que la formación especializada del profesorado de matemáticas en Panamá es una asignatura pendiente.

Para hacer frente a esta carencia, en 2017 se puso en marcha el Diplomado de Formación de Maestros de Matemáticas (Diplomado de aquí en adelante) en la Universidad de Panamá (MORALES MAURE; GARCÍA VÁSQUEZ; DURÁN GONZÁLEZ, 2019). Este programa es un referente de formación del profesorado de primaria de toda la República. Por él han pasado un total de 277 participantes, que supone prácticamente el $1.5 \%$ del total de maestros de primaria de la República de Panamá. Este Diplomado ha sido avalado con expertos a nivel internacional, y cuenta con colaboraciones de diversas universidades de todo el mundo (Figura $1)$.

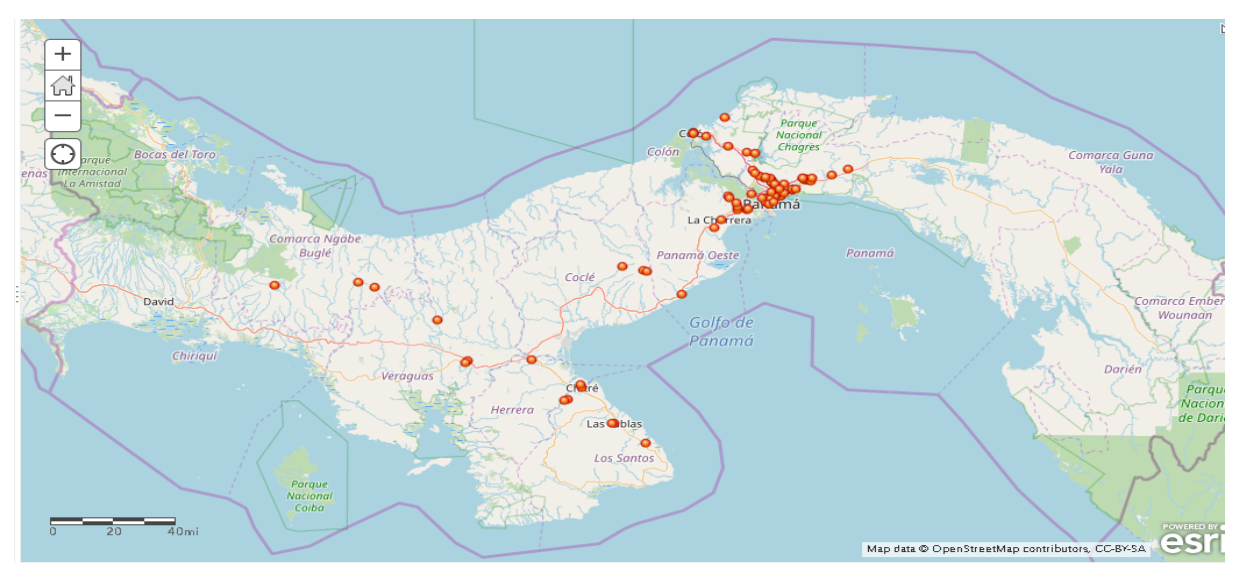

Figura 1 - Cobertura y alcance del Diplomado Fuente: ESRI - Panamá (2019)

En este artículo se evalúan las secuencias de aprendizaje realizadas por los maestros de primaria que han participado en el Diplomado, usando la herramienta denominada Criterios de Idoneidad Didáctica (CID, de aquí en adelante), desarrollada dentro del Enfoque Ontosemiótico (EOS) por Godino, Font y colaboradores (GODINO; BATANERO; FONT, 2019, 2020; BLANCO; GODINO; DIEGO-MANTECÓN, 2018), con la finalidad de determinar la calidad del Diplomado.

\section{Marco teórico}

Las investigaciones realizadas sobre el conocimiento y el desarrollo profesional de los 
profesores de matemáticas durante las últimas décadas sugieren que la formación profesional de los profesores de matemáticas tiene un claro impacto sobre la calidad de su actividad profesional (BREDA; PINO-FAN; FONT, 2017; PONTE, 2001; EVEN; BALL, 2009; LLINARES, 2013; SILVERMAN; THOMPSON, 2008).

El Diplomado que analizamos en este artículo toma como base teórica el enfoque Ontosemiótico (EOS). El EOS es un enfoque de investigación, dentro de la Didáctica de la Matemática, que integra elementos de diversas teorías de una manera original, y que está contribuyendo a la mejora del conocimiento de la enseñanza y aprendizaje de las matemáticas (GODINO; BATANERO; FONT, 2007, 2019).

Este enfoque supone la construcción de una teoría orientada a abordar, de manera sistemática, aspectos epistemológicos, ontológicos, semiótico-cognitivos y educativos de la enseñanza de las matemáticas (GODINO; BATANERO; FONT, 2020). El EOS parte de una visión antropológica y pragmatista de las matemáticas, consideradas como un campo del saber creado por las personas para resolver problemas. El EOS se asienta sobre tres principios epistemológicos principales (GODINO; BATANERO; FONT, 2019):

(1) La matemática es una actividad humana dirigida a resolver situaciones-problemas a través de sistemas de prácticas.

(2) Estas prácticas pueden ser de una persona, o compartidas en el seno de una institución.

(3) Para resolver problemas se articulan secuencias de prácticas, en un tiempo y lugar específicos, incluyendo diversos procesos y subprocesos (significación, conjeturación, representación, argumentación etc.).

Desde el EOS se centra la atención en las situaciones problema y las secuencias de prácticas que se usan para resolverlas, en las que participan objetos y procesos matemáticos, considerados entidades primarias, formando configuraciones de prácticas, objetos y procesos. Para comprender el conocimiento que se pone en juego en tales situaciones, es necesario elaborar herramientas que permitan tener en cuenta tales configuraciones.

Las configuraciones didácticas aparecen como unidades complejas de análisis de la práctica didáctica, a nivel micro. Se definen como segmentos de actividad didáctica que están delimitados por un principio y un final del proceso de resolución de la situación-problema. En la configuración didáctica participan los estudiantes, el docente, y todos los medios diseñados y/o implementados durante la sesión. El EOS estudia no solo los procesos cognitivos (idiosincráticos) de los estudiantes, sino también el marco institucional en el que se dan, a través de una mirada en la que se articulan los significados y objetos institucionales, con las respuestas 
idiosincráticas de los estudiantes. La complejidad de las configuraciones didácticas viene dada porque integran diferentes tipos de componentes, que permiten realizar una radiografía en detalle de la práctica matemática. Tal y como describen Godino y sus colaboradores (2020):

\begin{abstract}
En toda configuración didáctica ... se pueden diferenciar tres componentes: a) una configuración epistémica (sistema de prácticas, objetos y procesos matemáticos institucionales requeridos en la tarea), b) una configuración instruccional (sistema de funciones docentes, discentes y medios instruccionales que se utilizan, así como las interacciones entre los distintos componentes) y c) una configuración cognitivoafectiva (sistema de prácticas, objetos y procesos matemáticos personales que describe el aprendizaje y los componentes afectivos que le acompañan) (GODINO; BATANERO; FONT, 2020, p. 54).
\end{abstract}

El estudio de todos estos componentes se justifica desde la voluntad de mejora de la enseñanza y aprendizaje de las matemáticas. El EOS también se ha ocupado, específicamente, de esta finalidad a través del problema de la optimización del proceso de instrucción. Para ello, ha elaborado los denominados Criterios de Idoneidad Didáctica (CID).

Los CID son una herramienta de análisis cuyo objetivo es orientar y valorar la práctica docente del maestro de matemáticas (desde el diseño de la lección, hasta su evaluación, pasando por su implementación). Esta herramienta contiene seis criterios - o facetas, dependiendo del autor consultado, por ejemplo, en Esqué y Breda (2021) se usa criterio, y en Giacomone, Godino y Beltrán-Pellicer (2018) se usa faceta, que hacen referencia a diversos componentes presentes en la práctica de la enseñanza y aprendizaje de las matemáticas. A continuación de manera breve, se describen estos criterios:

a. Epistémico: es el grado de representatividad que tienen los significados institucionales implementados (o pretendidos), con relación a un significado global de referencia. El criterio epistémico busca revisiones de la adecuación del contenido matemático respecto a las situaciones-problemas, a los lenguajes, a las reglas (definiciones, propiedades, procedimientos), a los argumentos y a las relaciones entre ellos, utilizados para su enseñanza (CRUZ; GEA; GIACOMONE, 2017), y, según otros autores (BREDA, 2020), además de los aspectos comentados, este criterio busca evitar errores matemáticos y mejorar la riqueza de procesos. En pocas palabras, consiste en saber si lo que se enseña es una buena matemática.

b. Cognitivo: implica valorar la distancia razonable antes de iniciar el proceso de instrucción, es decir, valorar si lo que se quiere enseñar está a una distancia considerable de lo que saben los alumnos y, después del proceso, si los aprendizajes logrados se acercan a los que se pretendía enseñar (FONT; ADÁN; FERRERES, 2015). En otras palabras, consiste en saber cómo se transforman y modifican los conocimientos de los alumnos a partir de un nuevo conjunto de conceptos. 
c. Ecológico: se refiere a la adecuación al medio donde se desenvuelve el estudiante (currículum, lugar donde vive etc.). En ese sentido, Alsina y Domingo (2010, p. 12) sostienen que el criterio ecológico alude al grado en que un proceso de enseñanza y aprendizaje de las matemáticas resulta adecuado en el entorno donde se utiliza. El entorno incluye los componentes que influyen en la actividad que se lleva a cabo en el aula: tanto intrínsecos, como extrínsecos. En este último caso, se incluyen aspectos tales como el impacto de la familia sobre el aprendizaje y las pautas de trabajo, los referentes sociales y culturales (fundamentos de conocimiento) que emergen de la comunidad a la que pertenece el estudiante etc. (DIEZPALOMAR et al., 2014).

d. Mediacional: se refiere al uso de los recursos materiales y temporales a los que recurre el maestro y las situaciones que utiliza para contextualizar y motivar los conceptos matemáticos que se están trabajando en el aula. López (2016) afirma que el análisis del criterio mediacional se divide en tres aspectos: el tiempo, las condiciones particulares en que se da la clase y los recursos materiales que se usan en la enseñanza.

e. Afectivo: se refiere a fomentar la motivación que tiene el estudiante durante el proceso de aprendizaje. Este criterio está muy relacionado con las experiencias previas que ha tenido el estudiante durante su proceso de aprendizaje de las matemáticas. Por ello, es necesario que el docente sea capaz de promover los conocimientos a partir de situaciones didácticas que llamen su atención, generando un aprendizaje real. Este criterio tiene un claro impacto sobre la responsabilidad en el propio proceso de aprendizaje. Es decir, los alumnos estarán más interesados y dispuestos a resolver las tareas planteadas en la medida en que éstas les despierten emociones positivas (GODINO, 2011).

f. Interaccional: se refiere a la gestión de la comunicación que se produce entre los estudiantes entre sí, o entre estudiantes y maestro/a de matemáticas (u otros actores educativos que participen de la práctica de enseñanza y aprendizaje de las matemáticas). Uno de los principios fundamentales para la enseñanza de las matemáticas consiste en promover la interacción entre el alumnado durante la clase de matemáticas. Es fundamental comprender que los estudiantes no son receptores de una matemática acabada, sino que son agentes activos en el proceso enseñanza-aprendizaje, ya que ellos mismos desarrollan herramientas y comprensiones, y comparten sus experiencias unos con otros (GODINO, 2011).

Los CID se crean como un constructo para permitir a los [futuros] maestros reflexionar sobre su práctica, a fin de poder orientar su mejora a partir de sucesivos diseños y re-diseños de sus secuencias didácticas (FONT et al., 2018). Godino (2011) y sus colaboradores (FONT et al., 2018) propusieron usar metafóricamente la representación visual de esos CID como un 
hexágono (Figura 2). En los vértices de ese hexágono se sitúan cada uno de los criterios, y la distancia entre el centro y el vértice se divide en tres zonas, que corresponden con una presencia (o realización) baja, media o alta de cada uno de ellos. En una situación ideal, cada criterio debería estar presente a un nivel alto, de manera que obtendríamos un hexágono regular perfecto. Sin embargo, la realidad es que, en una situación de aula real encontraremos que se ha seguido cada criterio de manera desigual, con lo que obtendremos un hexágono irregular.

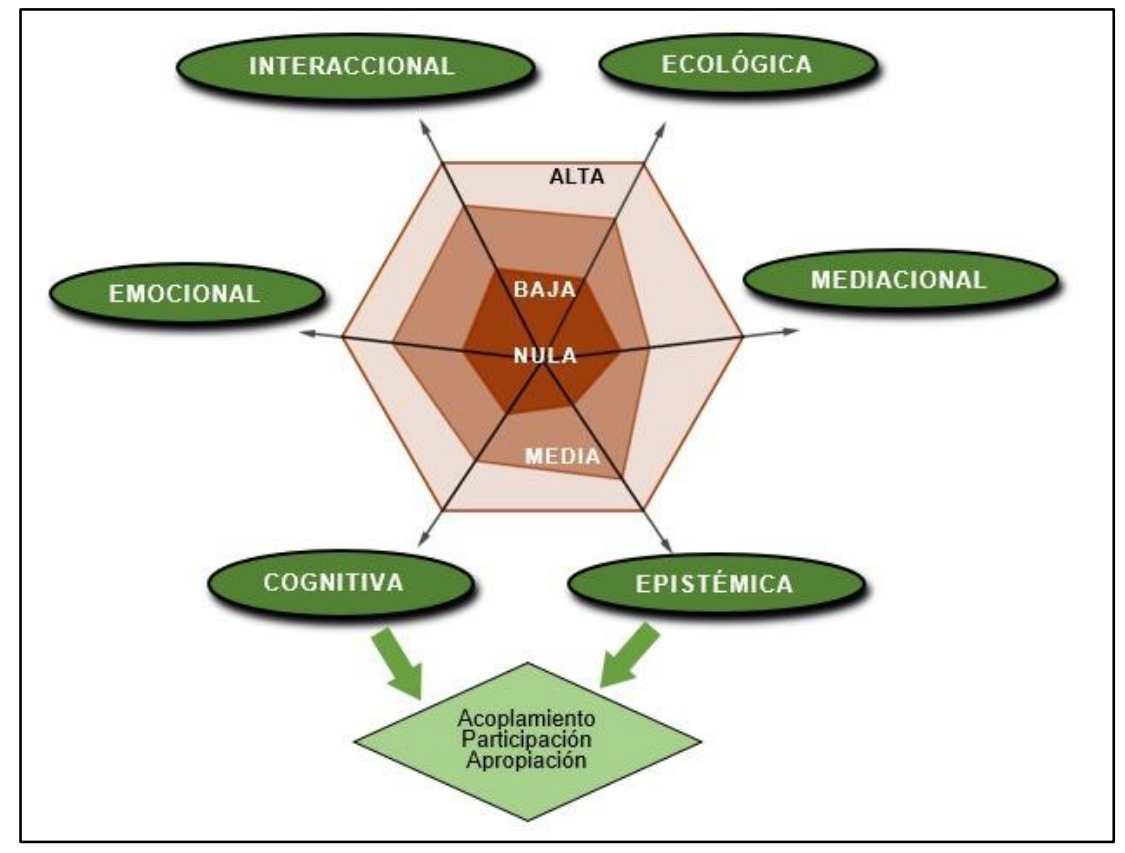

Figura 2 - Idoneidad didáctica - Adaptación Fuente: Godino (2013, p. 116)

La idoneidad didáctica depende del grado de adecuación que tiene el peso dado a cada criterio, teniendo en cuenta el contexto donde trabaja el maestro, por lo cual todo docente debe tomar una postura de equilibrista (FONT, 2019), tal y como dice Seckel (2015). Con esta metáfora, Seckel recoge el testimonio de un maestro que reflexiona cómo los criterios CID le sirven al maestro de matemáticas para conseguir impartir una clase idónea. Pero, para ello tiene que ser casi como un malabarista o equilibrista: tiene que ser capaz de encontrar el equilibrio entre los pesos dados a cada criterio.

En un inicio, los CID no se propusieron como una herramienta para medir la idoneidad de un proceso de instrucción; más bien se pensaron como una herramienta cualitativa que podía ser útil al profesor para reflexionar sobre su práctica, o bien como categorías a priori para inferir criterios que orientan la práctica del profesor en servicio o en formación (por ejemplo, en SECKEL et al., 2019). Pero, en trabajos posteriores se ha ido viendo el potencial que tiene este constructo no solo como herramienta de reflexión, sino para evaluar tanto las prácticas de aula, como los diseños y re-diseños docentes (e.g. secuencias didácticas). 


\section{Metodología}

El diseño de la investigación parte de la elaboración de secuencias didácticas de los futuros maestros/as y maestros/as en servicio participantes en el Diplomado de Formación de Maestros de Matemáticas de la Universidad de Panamá. Cada maestro/a participante en el Diplomado tiene que elaborar una secuencia didáctica. Esa secuencia se re-diseña posteriormente, de acuerdo con los aprendizajes adquiridos a lo largo de las diferentes sesiones del Diplomado. Finalmente, cada participante tiene que implementar la secuencia, y grabar en video dicha implementación. Posteriormente, la grabación se sube a una plataforma virtual, donde los y las participantes también han ubicado tanto su primera secuencia didáctica, como los re-diseños posteriores. La Figura 3 ilustra cómo se ha planteado este diseño metodológico.

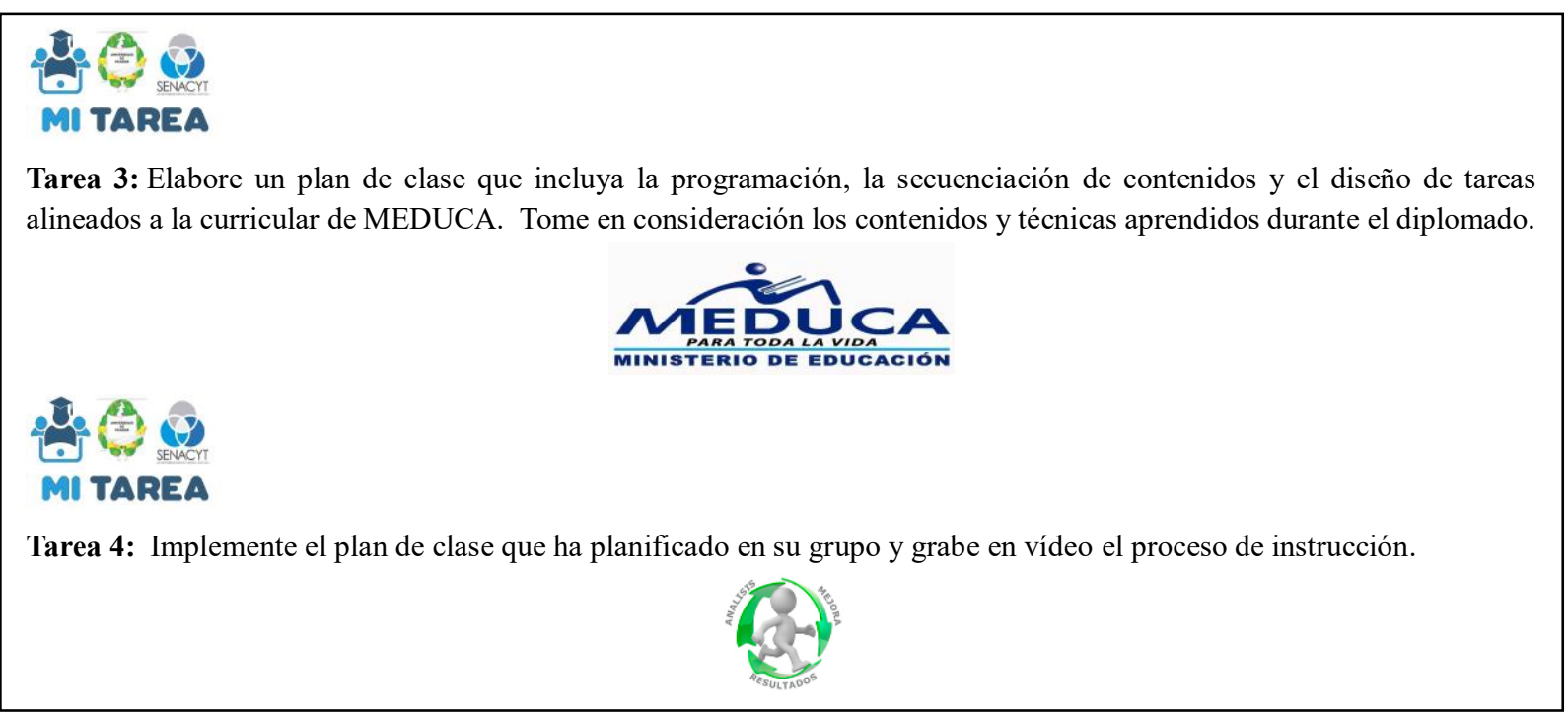

Figura 3 - Planteamiento de las tareas a los/as participantes en el Diplomado Fuente: Campus Virtual - Universidad de Panamá (2019)

La unidad básica de información que se ha recabado en el trabajo de campo han sido videos de secuencias didácticas (clases) desarrolladas por los y las participantes en el Diplomado. El análisis de videos ha sido ya usado, previamente, en el ámbito de la investigación de la didáctica de la matemática (MORALES-LÓPEZ; FONT, 2019; PLANAS, 2006). Los videos permiten aproximarse a la complejidad inherente en la impartición de una lección de matemáticas en el aula. También, permiten observar cómo los futuros/as docentes y maestros/as en servicio participantes en el estudio han (o no) adquirido los aprendizajes del Diplomado.

Por otro lado, también hemos dispuesto de las planificaciones docentes (secuencias didácticas) elaboradas por los futuros maestros y maestras y maestros/as en servicio que han participado en la investigación, así como los rediseños que han elaborado a lo largo de su participación en el Diplomado. Estos rediseños se han plasmado en la grabación de video, que 
ha sido el trabajo final presentado por los futuros/as maestros/as y maestros/as en servicio en el Diplomado.

Han participado un total de 93 futuros maestros/as y maestros/as en servicio, de entre las que se ha seleccionado una muestra de diez maestros/as, tal como se muestra en la Tabla 1. Para seleccionar a esos/as diez maestros/as se tuvo en cuenta el criterio de los años de servicio, para garantizar tanto tener a maestros/as con una larga experiencia docente, como maestros/as nuevos/as, con poca o ninguna experiencia docente.

\begin{tabular}{cc} 
Tabla 1 - Maestros/as participantes en el trabajo de campo & ${ }^{1}$ \\
\hline Participante & Años de servicio \\
\hline Carmela & 22 \\
Zaida & 20 \\
Yina & 16 \\
Lucía & 14 \\
Rubén & 13 \\
Guadalupe & 9 \\
Miriam & 6 \\
Lina & 4 \\
Oris & 0 \\
Mercedes & 0 \\
\hline
\end{tabular}

Fuente: elaboración propia

En el análisis que usamos en este estudio se adopta un enfoque metodológico basado en la mirada del investigador, tal y como la definen Lerman (2001) y Planas (2006). Los videos han sido visualizados y discutidos conjuntamente con expertos, para la selección de clips significativos de acuerdo con el marco de análisis presentado (los CID), aplicando el procedimiento de análisis descrito por Pirie (1998).

De esa manera, primero se transcribieron los videos y se codificaron usando una tabla de códigos creados a partir de la operacionalización de los CID (ver Cuadro 1). Se usó la definición de este constructo que dan Breda, Font y Pino-Fan (2018), quienes desglosan los CID en componentes e indicadores. De acuerdo con investigaciones previas, los CID permiten evaluar/analizar el grado de madurez que tienen los maestros/as al desarrollar sus secuencias didácticas cuando las implementan en su salón de clase. Tomando como base la versión que presentan Breda, Font y Pino-Fan (2018) de los CID desglosados por componentes e indicadores, se creó una tabla de códigos, usando una escala de Likert para concretar los indicadores que presentan Breda, Font y Pino-Fan (2018), en una escala con valores que van desde cero hasta tres, tal y como se muestra en el Cuadro 1, adjunto.

\footnotetext{
1 Todos los nombres son pseudónimos, para preservar el anonimato de las personas participantes.
} 


\begin{tabular}{|c|c|c|c|}
\hline Criterio & Componente & Código & Indicadores \\
\hline \multirow{4}{*}{ 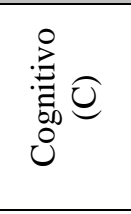 } & 1. Conocimientos previos & $\mathrm{C} 1$ & $0=$ Nulos, $1=$ Pobres $2=$ Bastantes, $3=$ Exhaustivos \\
\hline & $\begin{array}{l}\text { 2. Adaptación curricular a las } \\
\text { diferencias individuales }\end{array}$ & $\mathrm{C} 2$ & $\begin{array}{l}0=\text { No hay, } 1=\text { Hay poca adaptación, } \\
2=\text { Casi siempre hay }, 3=\text { Siempre hay }\end{array}$ \\
\hline & 3. Aprendizaje & $\mathrm{C} 3$ & $0=$ Nulo, $1=$ Bajo, $2=$ Medio, $3=$ Alto \\
\hline & 4. Demanda cognitiva & $\mathrm{C} 4$ & $0=$ Nula, $1=$ Baja, $2=$ Media, $3=$ Alta \\
\hline \multirow{4}{*}{ 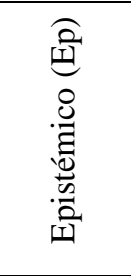 } & 1. Errores & Ep1 & $\begin{array}{l}0=\text { Siempre hay, } 1=\text { Casi siempre, } \\
2=\text { Hay pocos, } 3=\text { No hay }\end{array}$ \\
\hline & 2. Ambigüedades & Ep2 & $\begin{array}{l}0=\text { Siempre hay }, 1=\text { Casi siempre, } \\
2=\text { Hay pocas, } 3=\text { No hay }\end{array}$ \\
\hline & 3. Riquezas de procesos & Ep3 & $\begin{array}{l}0=\text { No hay, } 1=\text { Hay poca, } \\
2=\text { Casi siempre, } 3=\text { Siempre hay }\end{array}$ \\
\hline & 4. Representatividad de la complejidad & Ep4 & $0=$ Nula, $1=$ Baja, $2=$ Media, $3=$ Alta \\
\hline \multirow{4}{*}{ 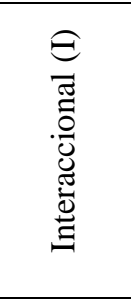 } & 1. Interacción docente - discente & I1 & $\begin{array}{l}0=\text { No hay, } 1=\text { Hay poca interacción, } \\
2=\text { Casi siempre, } 3=\text { Siempre hay }\end{array}$ \\
\hline & 2. Interacción entre discentes & $\mathrm{I} 2$ & $\begin{array}{l}0=\text { No hay, } 1=\text { Hay poca interacción, } \\
2=\text { Casi siempre, } 3=\text { Siempre hay }\end{array}$ \\
\hline & 3. Autonomía & $\mathrm{I} 3$ & $\begin{array}{l}0=\text { No hay, } 1=\text { Hay poca autonomía, } \\
2=\text { Casi siempre, } 3=\text { Siempre hay }\end{array}$ \\
\hline & 4. Evaluación formativa & $\mathrm{I} 4$ & $\begin{array}{l}0=\text { No hay, } 1=\text { Hay poca evaluación, } \\
2=\text { Casi siempre, } 3=\text { Siempre hay }\end{array}$ \\
\hline \multirow{3}{*}{ 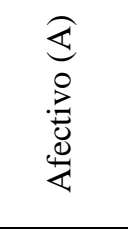 } & $\begin{array}{l}\text { 1. Actitudes (perseverancia, } \\
\text { responsabilidad, implicación) }\end{array}$ & A1 & $\begin{array}{l}0=\text { No hay, } 1=\text { Hay poca, } \\
2=\text { Casi siempre, } 3=\text { Siempre hay }\end{array}$ \\
\hline & 2. Intereses y necesidades & $\mathrm{A} 2$ & $\begin{array}{l}0=\text { No se valoran, } 1=\text { Se valoran poco, } \\
2=\text { Se valoran casi siempre, } 3=\text { Siempre se valoran }\end{array}$ \\
\hline & 3. Emociones positivas & A3 & $\begin{array}{l}0=\text { No se promueven, } 1=\text { Se promueven poco, } \\
2=\text { Casi siempre se promueven, } 3=\text { Se promueven siempre }\end{array}$ \\
\hline \multirow{4}{*}{ 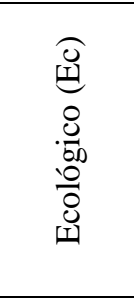 } & 1. Adaptación al currículum & Ec1 & $\begin{array}{l}0=\text { No hay, } 1=\text { Hay poca adaptación, } \\
2=\text { Casi siempre hay, } 3=\text { Siempre hay }\end{array}$ \\
\hline & 2. Conexiones intra e interdisciplinares & Ec2 & $\begin{array}{l}0=\text { No hay, } 1=\text { Hay pocas } \\
2=\text { Casi siempre hay, } 3=\text { Siempre hay }\end{array}$ \\
\hline & 3. Utilidad socio-laboral & Ec3 & $\begin{array}{l}0=\text { No hay, } 1=\text { Hay poca, } \\
2=\text { Casi siempre hay, } 3=\text { Siempre hay }\end{array}$ \\
\hline & 4. Innovación didáctica & Ec4 & $\begin{array}{l}0=\text { No hay, } 1=\text { Hay poca, } \\
2=\text { Casi siempre hay, } 3=\text { Siempre hay }\end{array}$ \\
\hline \multirow{5}{*}{ 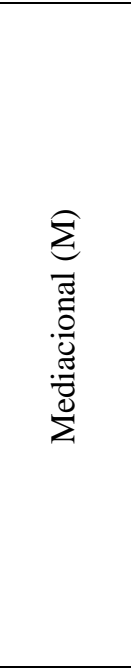 } & $\begin{array}{l}\text { 1. Recursos materiales (manipulativos, } \\
\text {...) }\end{array}$ & M1 & $\begin{array}{l}0=\text { No se utilizan, } 1=\text { Se utilizan poco, } \\
2=\text { Se utilizan casi siempre, } 3=\text { Se utilizan siempre }\end{array}$ \\
\hline & $\begin{array}{l}\text { 2. Agrupación de Alumnos (grupos } \\
\text { pequeños o todos los alumnos) }\end{array}$ & M2 & $\begin{array}{l}0=\text { De manera incorrecta de acuerdo con la tarea, } \\
1=\text { De manera poco correcta } \ldots, 2=\text { Casi siempre están } \\
\text { agrupados de manera correcta ..., 3= Siempre están } \\
\text { agrupados de manera correcta }\end{array}$ \\
\hline & $\begin{array}{l}\text { 3. Horario (actividad que se propone } \\
\text { tiempo límite o es abierta) }\end{array}$ & M3 & $\begin{array}{l}0=\text { No se ajusta a la necesidad de la tarea, } 1=\text { Se ajusta } \\
\text { poco a la necesidad de la tarea, } 2=\text { Casi siempre se ajusta } \\
\text { a la necesidad de la tarea, } 3=\text { se ajusta totalmente a la } \\
\text { necesidad de la tarea }\end{array}$ \\
\hline & $\begin{array}{l}\text { 4. Condiciones del aula (Existen } \\
\text { recursos para los procesos) }\end{array}$ & M4 & $\begin{array}{l}0=\text { No son adecuadas a las necesidades de la tarea, } \\
1=\text { Son poco adecuadas a las necesidades... } \\
2=\text { Casi siempre son adecuadas...., } \\
3=\text { Son totalmente adecuadas a las necesidades }\end{array}$ \\
\hline & $\begin{array}{l}\text { 5. Distribución del Tiempo (de la } \\
\text { enseñanza colectiva / tutorización } \\
\text { tiempo de aprendizaje) }\end{array}$ & M5 & $\begin{array}{l}0=\text { No es adecuado a las necesidades de la tarea, } \\
1=\text { Es poco adecuado a las necesidades de la tarea, } \\
2=\text { Casi siempre es adecuado a las necesidades ..., } \\
3=\text { Siempre es adecuado a las necesidades de la tarea. }\end{array}$ \\
\hline
\end{tabular}

Cuadro 1 - Ponderaciones de los Criterios de la Idoneidad didáctico-matemática Fuente: elaboración propia

Cada uno de los seis criterios está desglosado en componentes (BREDA; FONT; PINOFAN, 2018). A cada componente se le asigna un código. Esos componentes, se cuantifican usando una escala de Likert, donde se considera si dicho componente está presente (completamente, casi completamente, o poco presente), o no lo está. Se utilizan los valores del 
cero al tres para indicar cada uno de estos cuatro estados posibles.

A partir de las categorías (componentes e indicadores) descritos en el Cuadro 1, se realizó un análisis de contenido de las transcripciones de las grabaciones de video. Como unidad de análisis se definió la frase, o frases, que semánticamente hacen referencia a una idea o concepto (que puede estar expresado en una sola frase, o en un conjunto de frases subordinadas unas a las otras, semánticamente hablando). Estas ideas o conceptos se codificaron usando las categorías descritas en el Cuadro 1.

Se usó el EXCEL para realizar este análisis, poniendo los fragmentos de la transcripción en filas (una fila por cada idea o concepto) y las categorías de análisis (componentes) en las columnas. Se usaron los números $0,1,2$ y 3 para denotar los indicadores correspondientes a cada componente. Al final de cada transcripción (correspondiente al vídeo de cada maestro/a participante en el estudio), se sumaron las puntuaciones obtenidas en cada uno de los componentes. De ese modo, se han definido dos índices: el índice Criterio y el índice CID.

El índice Criterio se define como el sumatorio de los indicadores correspondientes a cada uno de los componentes, dividido entre los componentes. Por ejemplo, para el caso del criterio epistémico, se ha procedido a sumar los cuatro componentes, por un lado; y la suma total de los indicadores de dichos componentes, por el otro. A continuación, se procede a dividir el sumatorio de los indicadores del componente, entre los cuatro componentes. El resultado es un número comprendido entre 0 y 3 , que describe el cómputo promedio en el que ese componente se cumple (o no). De manera general, el índice Criterio se describe como:

Fórmula 1. IC $=\frac{\sum I}{\sum C}$

siendo IC el índice Criterio, $\Sigma \mathrm{I}$ el valor del sumatorio de los indicadores, y $\Sigma \mathrm{C}$ la cantidad de componentes del criterio respectivo. Existe un IC para cada una de los seis criterios (e.g. $\mathrm{IC}_{\mathrm{ep}}$ para el criterio epistémico; $\mathrm{IC}_{\mathrm{c}}$ para el cognitivo etc.).

El índice CID se define como la razón del sumatorio del valor alcanzado en cada uno de los IC. En términos generales, el índice $C I D$ se describe como:

Fórmula 2. ICID $=\frac{\sum I C_{e p}+I C_{c}+I C_{i}+I C_{a}+I C_{e c}+I C_{m}}{6}$

El resultado es un número que se encuentra entre 0 y 3 , que describe si esa unidad didáctica, secuencia, práctica o actividad es (o no) idonea en términos didácticos.

Para cada uno de los videos seleccionados, se realizó un análisis de acuerdo con el procedimiento explicado arriba. Para garantizar la fiabilidad del análisis, cada codificación se sometió a discusión entre dos de los investigadores firmantes de este artículo. En el caso de no existir acuerdo en la codiciación, se recurrió al juicio de expertos para decidir la codificación 
definitiva.

El Cuadro 2 ilustra el proceso de análisis realizado, a título de ejemplo.

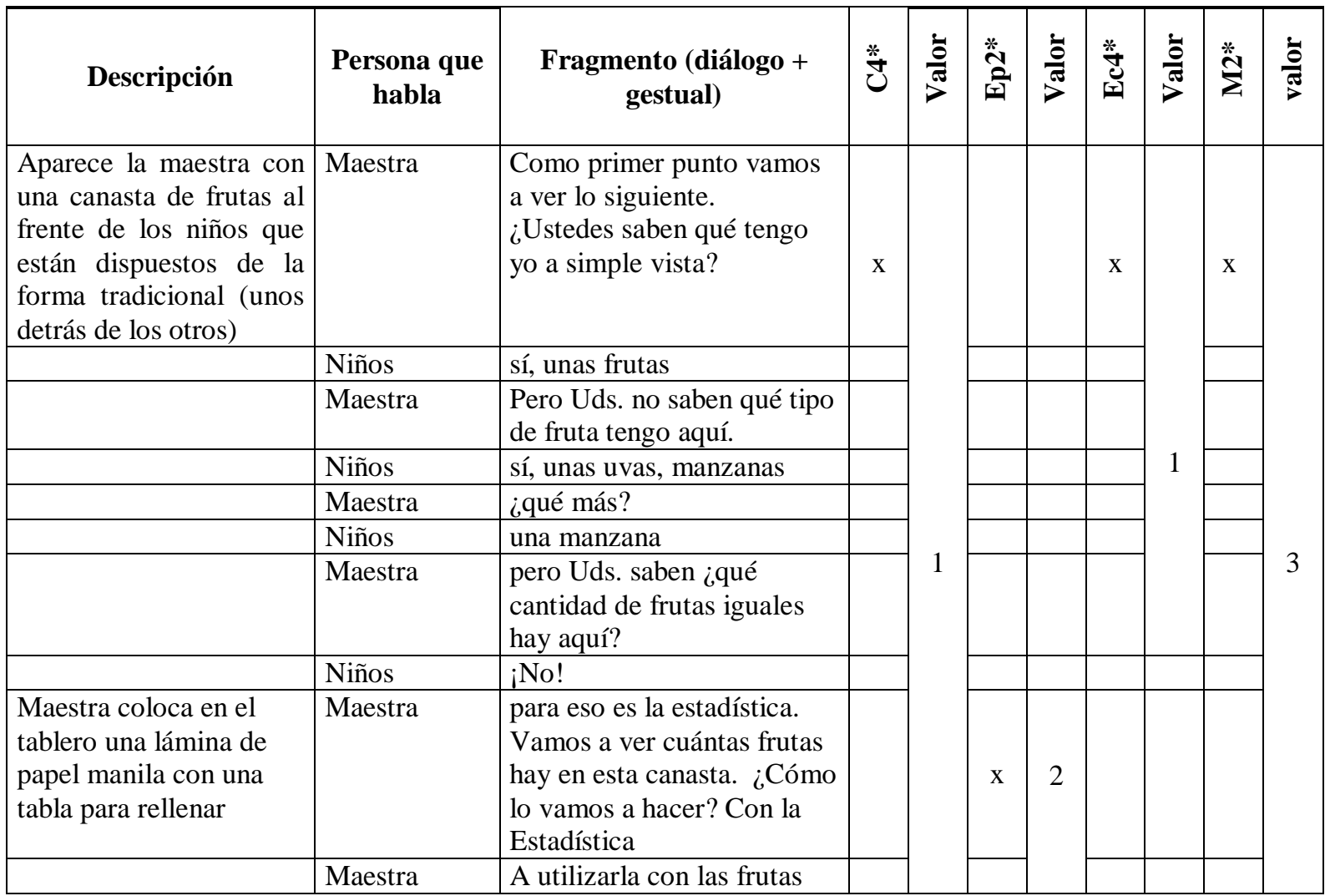

Cuadro 2 - Transcripción y valoración de los fragmentos de diálogo de la transcripción del video-clase de la maestra Oris

Fuente: elaboración propia

Se observa que cada uno de los componentes que definen, respectivamente, los seis criterios es ponderado usando los valores de la escala de Likert, tal y como se ha mencionado más arriba. Cuando uno de los componentes está presente completamente en la secuencia didáctica, se codifica con el código del componente, y se le asigna valor 3 ; en cambio, cuando ese componente no aparece en el fragmento de transcripción, entonces se codifica con el código correspondiente al valor 0 . En el ejemplo que se ilustra en el Cuadro 2, el código $\mathrm{C} 4$ se refiere al cuarto componente del criterio cognitivo: Demanda cognitiva. Cuando la maestra realiza la pregunta Como primer punto vamos a ver lo siguiente: ¿Ustedes saben qué tengo yo a simple vista? podemos comprobar que existe una pobre Demanda cognitiva (por lo menos, desde el punto de vista del aprendizaje de las matemáticas); en la línea de transcripción, cuando los niños responden Sí, unas frutas, se confirma que no hay una gran exigencia cognitiva en dicha respuesta (por tanto, el valor que se le asigna es de 1, es decir, hay una baja demanda cognitiva). Este procedimiento de análisis se repite para cada una de las intervenciones en las transcripciones de los datos recabados.

A continuación, debido a que cada criterio se desglosa en varios componentes (BREDA; 
FONT; PINO-FAN, 2018), se hace un promedio para obtener el valor final asignado a cada una de los criterios. En la Tabla 2 se presenta el caso del índice Criterio interaccional, a título de ejemplo, también para el caso de la maestra Oris.

Tabla 2 - Tabla de valoración del Criterio Interaccional de la clase de la maestra Oris

\begin{tabular}{ccccc}
\hline Criterio & $\begin{array}{c}\text { Componente } \\
\text { Codificado }\end{array}$ & $\mathrm{I}$ & $\mathrm{C}$ & $\mathrm{IC}_{\mathrm{i}}$ \\
\hline Interaccional & $\mathrm{I} 1$ & 3 & 1 & \\
(I) & $\mathrm{I} 2$ & 3 & 1 & \\
& $\mathrm{I} 3$ & 3 & 1 & \\
& $\mathrm{I} 4$ & 0 & 1 & \\
& Total & 9 & 4 & 2.25 \\
\hline
\end{tabular}

Fuente: elaboración propia

Este proceso se repite para cada una de los seis criterios, con lo cual se obtiene, de esta manera, una puntuación correspondiente a cada una ellos usando la fórmula 1. La Tabla 3 ilustra el caso de las puntuaciones obtenidas por el análisis del video de la maestra Oris para cada uno de los seis criterios.

Finalmente, usando la fórmula 2 se calcula el ICID correspondiente a cada una de las secuencias didácticas de cada persona que ha participado en este estudio (en el caso de nuestro ejemplo, la maestra Oris). El rango del ICID está entre 0 y 3; indicando que si la secuencia didáctica de la maestra/o obtiene un ICID igual a 0 quiere decir que no existe idoneidad didáctica en su secuencia didáctica, mientras que si su ICID es igual a 3, quiere decir que la idoneidad didáctica de su secuencia didáctica es alta.

Tabla 3 - Evaluación de la Idoneidad Didactico Matemática de la secuencia elaborada por la maestra Oris

\begin{tabular}{ccc}
\hline Criterio & IC & ICID \\
\hline Cognitivo (C) & 1.50 & \\
Epistémico (Ep) & 2.25 & \\
Interaccional (I) & 2.25 & \\
Afectivo (A) & 2.00 & \\
Ecológico (Ec) & 0.75 & \\
Mediacional (M) & 3.00 & 1.96 \\
\hline Total & 11.75 &
\end{tabular}

Fuente: elaboración propia

Par responder al objetivo que nos hemos propuesto en este artículo, sobre la evaluación de las secuencias didácticas de los y las participantes en el Diplomado, se considera que un ICID igual o superior a 1.5 indica que existe una idoneidad razonablemente adecuada, mientras que un ICID inferior a 1.5 sugiere que esta idoneidad no es suficiente. 


\section{Resultados}

A continuación se presentan los resultados obtenidos en este estudio. Los resultados preliminares sugieren que los CID permiten identificar aspectos relevantes en las lecciones analizadas de los diez maestros seleccionados.

La Tabla 4 muestra los resultados obtenidos por las secuncias didácticas de los diez maestros que participaron en el estudio, por lo que respecta al índice CID.

Tabla 4 - Puntuaciones del índice CID obtenidas por las secuncias didácticas de los maestros

\begin{tabular}{cc}
\multicolumn{3}{c}{ participantes } \\
\hline Participante & ICID \\
\hline Carmela & 2.58 \\
Zaida & 2.17 \\
Yina & 2.46 \\
Lucía & 2.25 \\
Rubén & 2.33 \\
Guadalupe & 2.46 \\
Miriam & 1.79 \\
Lina & 1.84 \\
Oris & 1.96 \\
Mercedes & 2.10 \\
\hline
\end{tabular}

Fuente: elaboración propia

Como podemos observar, en todos los casos el índice CID está por encima del 1.5, considerado el umbral a partir del cual se puede considerar que el material analizado presenta una cierta idoneidad didáctica. Este valor se sitúa justo en medio del rango 0-3, con el que se mide cada una de las idoneidades. La mitad de los maestros y maestras que participaron en el estudio presentan una puntuación que se sitúa entre el 2.6 y el 1.7, aproximándose a lo que podría considerarse una idoneidad aceptable por lo que respecta a las secuencias didácticas que han diseñado e implementado.

Si observamos estos resultados, desde el punto de vista de la experiencia docente que tiene el maestro/a participante en el estudio, observamos que aparece una regularidad que es destacable: a más años de experiencia docente, mayor es el índice CID obtenido, cosa que sugiere que existe una cierta relación entre la experiencia docente (medida en años), y la capacidad para diseñar secuencias didácticas idóneas, desde el punto de vista del análisis planteado por el EOS. En la Tabla 5 se pueden apreciar mejor estos resultados.

Tabla 5 - Puntuaciones del índice CID obtenidas por los maestros y maestras participantes en el estudio, según su experiencia docente en años

\begin{tabular}{ccc}
\hline Participante & Años de experiencia docente & ICID \\
\hline Carmela & 22 & 2.58 \\
Zaida & 20 & 2.17 \\
Yina & 16 & 2.46 \\
Lucía & 14 & 2.25 \\
Rubén & 13 & 2.33 \\
Guadalupe & 9 & 2.46
\end{tabular}




\begin{tabular}{ccc} 
Miriam & 6 & 1.79 \\
Lina & 4 & 1.84 \\
Oris & 0 & 1.96 \\
Mercedes & 0 & 2.10 \\
\hline \multicolumn{2}{l}{}
\end{tabular}

Las puntuaciones obtenidas por cada una de las maestras y maestros que han participado en el estudio han sido representadas gráficamente mediante el hexágono utilizado por Godino y sus colaboradores para visualizar de manera global la idoneidad didáctica (GODINO; BATANERO; FONT, 2019, 2020; BLANCO; GODINO; DIEGO-MANTECÓN, 2018). Sin embargo, en nuestro caso, más allá de una metáfora es una herramienta metodológica de análisis puesto que el hexágono se construye a partir de las puntuaciones obtenidas en cada uno de los criterios, tal y como se ha explicado en el apartado de metodología. Eso nos permite obtener una imagen comparable de cada uno de los sujetos (ver Figuras 4, 5, 6, 7, 8, 9, 10, 11, 12 y 13).

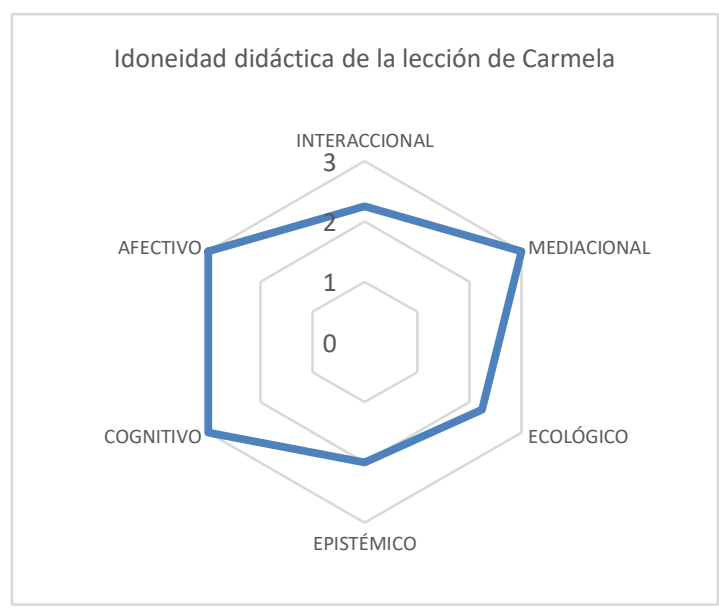

Figura 4 - Hexágono del caso de la maestra Carmela Fuente: elaboración propia

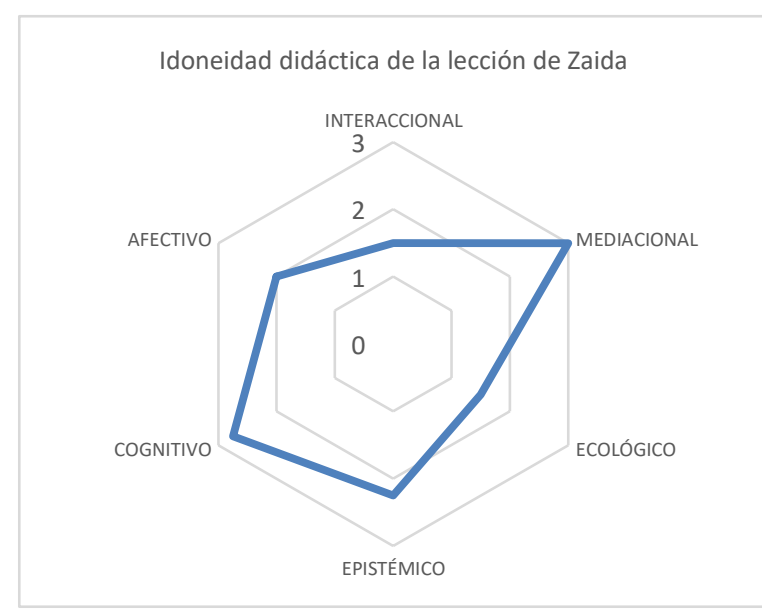

Figura 5 - Hexágono del caso de la maestra Zaida Fuente: elaboración propia 
Idoneidad didáctica de la lección de Yina

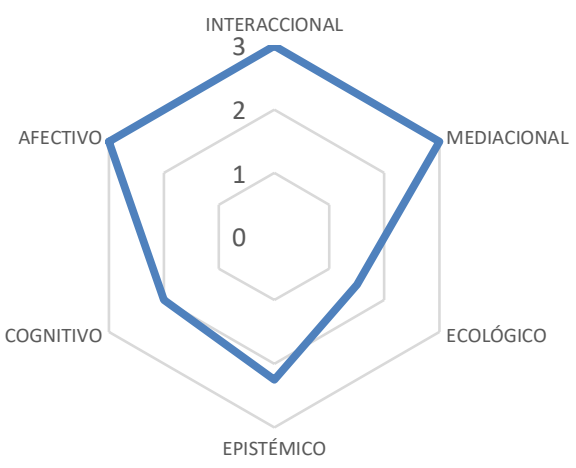

Figura 6 - Hexágono del caso de la maestra Yina Fuente: elaboración propia

Idoneidad didáctica de la lección de Rubén

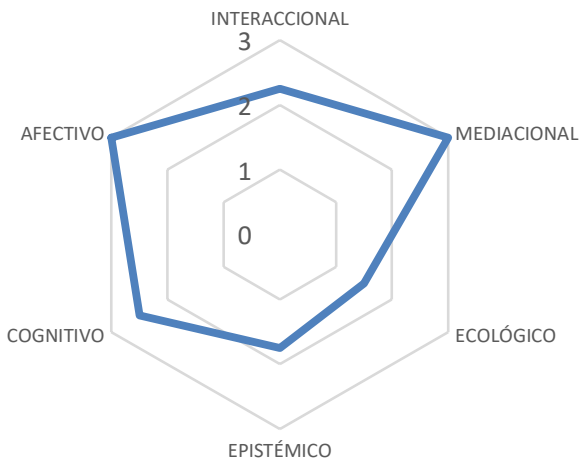

Figura 8 - Hexágono del caso del maestro Rubén Fuente: elaboración propia

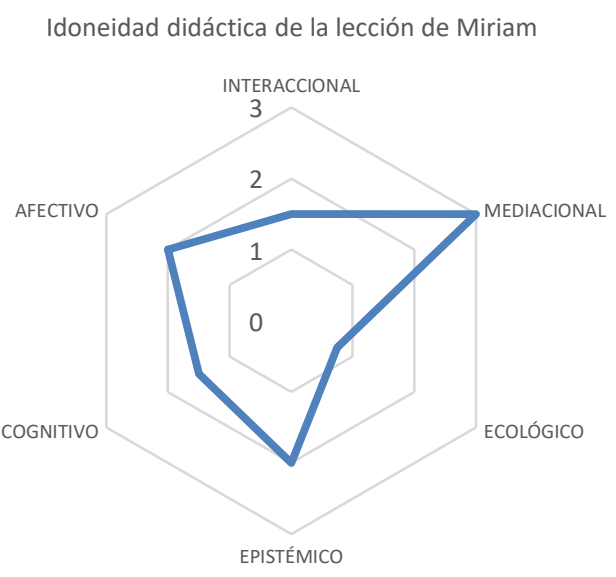

Figura 10 - Hexágono del caso de la maestra Miriam Fuente: elaboración propia

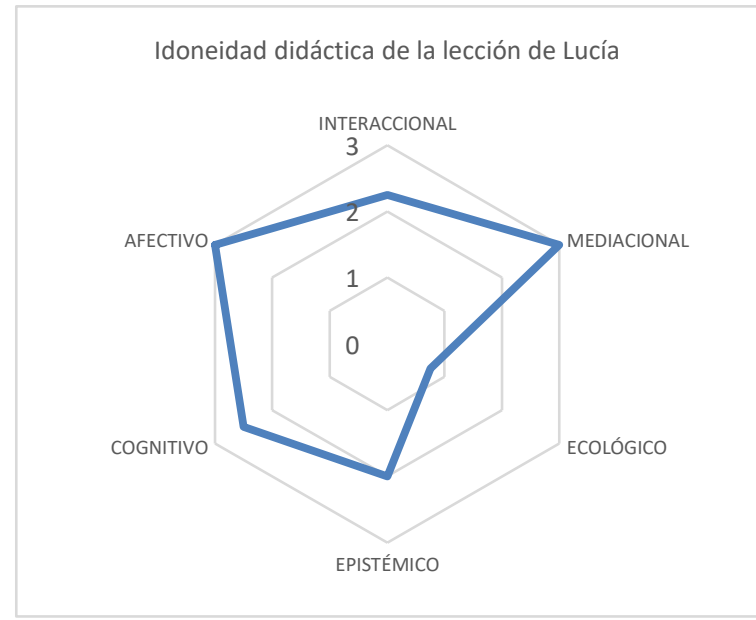

Figura 7 - Hexágono del caso de la maestra Lucía Fuente: elaboración propia

Idoneidad didáctica de la lección de Guadalupe

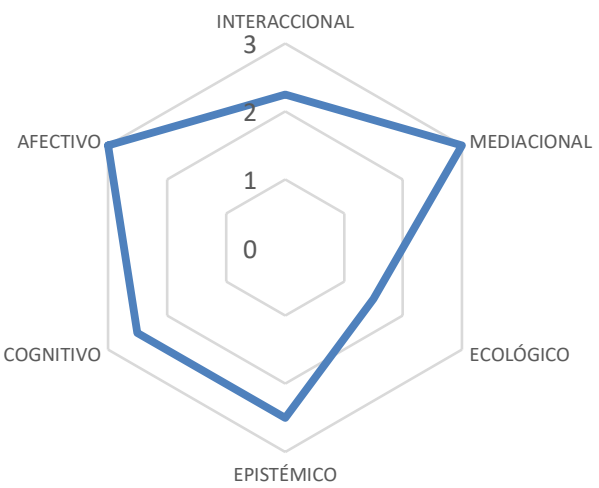

Figura 9 - Hexágono del caso de la maestra Guadalupe Fuente: elaboración propia

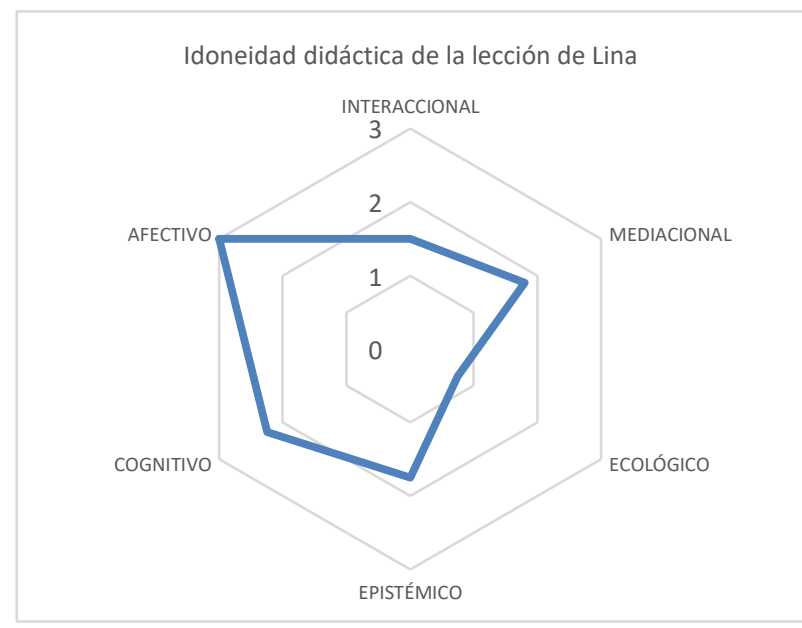

Figura 11 - Hexágono del caso de la maestra Lina Fuente: elaboración propia 


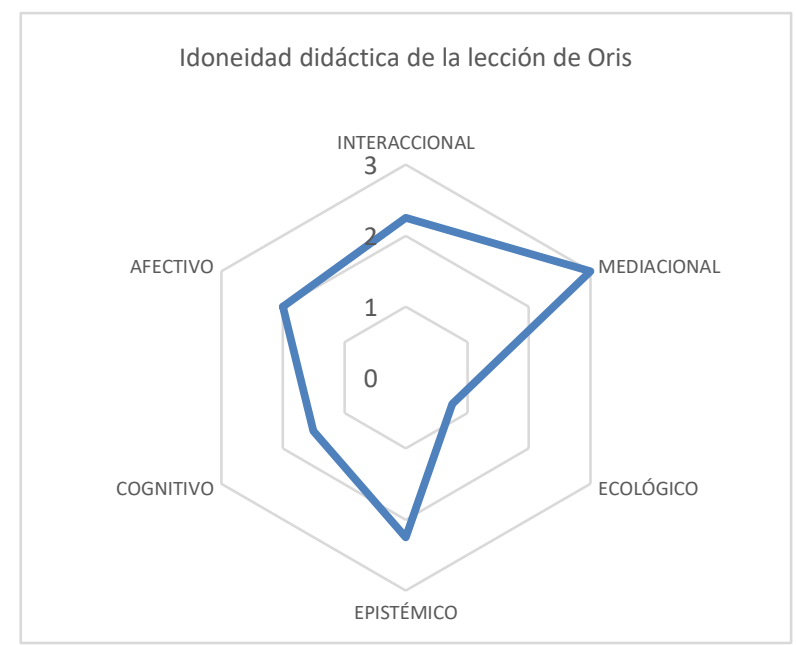

Figura 12 - Hexágono del caso de la maestra Oris Fuente: elaboración propia

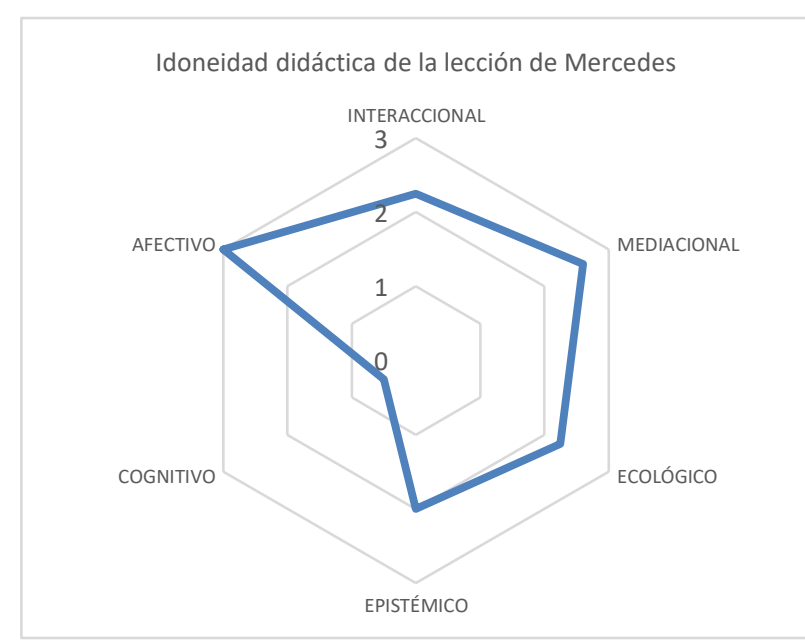

Figura 13 - Hexágono del caso de la maestra Mercedes Fuente: elaboración propia

Como se puede observar, las cuatro maestras con menos experiencia docente son las que obtienen puntuaciones más bajas en el índice CID. Ese resultado sugiere que la experiencia puede estar jugando un papel relevante en el diseño y planificación de los maestros y de las maestras, que lejos de adquirir vicios o tender a usar siempre las mismas estrategias o recursos, integran prácticas y maneras de hacer en sus diseños didácticos que funcionan (y que desde el punto de vista del análisis de su idoneidad didáctica, resultan tender a una idoneidad óptima en el sentido de puntuar alto en cada uno de los CID).

Para contrastar esta última afirmación, hemos hecho un análisis por criterios para comprobar si el índice CID corresponde con una cierta homogeneidad en la puntuación desglosada por criterios, o encontramos diferencias notables a destacar. La Tabla 6 presenta los resultados desglosados por criterios para los maestros y las maestras participantes en el estudio.

Tabla 6 - Valoraciones por criterio de las secuencias didácticas de los maestros participantes

\begin{tabular}{|c|c|c|c|c|c|c|c|c|}
\hline \multicolumn{9}{|c|}{ en el estudio } \\
\hline Participante & $\mathrm{IC}_{\mathrm{ec}}$ & $\mathrm{IC}_{\mathrm{ep}}$ & $\mathrm{IC}_{\mathrm{c}}$ & $\mathrm{IC}_{\mathrm{a}}$ & $\mathrm{IC}_{\mathrm{i}}$ & $\mathrm{IC}_{\mathrm{m}}$ & ICID & Desviación \\
\hline Carmela & 2.25 & 2.0 & 3 & 3 & 2.25 & 3 & 2.58 & 0.28 \\
\hline Zaida & 1.5 & 2.25 & 2.75 & 2 & 1.5 & 3 & 2.17 & 0.26 \\
\hline Yina & 1.5 & 2.25 & 2.0 & 3 & 3 & 3 & 2.46 & 0.27 \\
\hline Lucía & 0.75 & 2.0 & 2.5 & 3 & 2.25 & 3 & 2.25 & 0.26 \\
\hline Rubén & 1.5 & 1.75 & 2.5 & 3 & 2.25 & 3 & 2.33 & 0.26 \\
\hline Guadalupe & 1.5 & 2.5 & 2.5 & 3 & 2.25 & 3 & 2.46 & 0.27 \\
\hline Miriam & 0.75 & 2 & 1.5 & 2 & 1.5 & 3 & 1.79 & 0.29 \\
\hline Lina & 0.75 & 1.75 & 2.25 & 3 & 1.5 & 1.8 & 1.84 & 0.28 \\
\hline Oris & 0.75 & 2.25 & 1.5 & 2 & 2.25 & 3 & 1.96 & 0.27 \\
\hline Mercedes & 2.25 & 2 & 0.5 & 3 & 2.25 & 2.6 & 2.1 & 0.26 \\
\hline
\end{tabular}

Como se puede observar en la Tabla 6, no existe una gran dispersión en la distribución de puntuaciones por criterios, para cada uno de los maestros y de las maestras. Es relevante 
destacar que en el caso de Lina y de Mercedes (ambas maestras con reducida o inexistente experiencia docente) encontramos mayores diferencias en las puntuaciones entre criterios. Pero esto también ocurre en los casos de Yina y de Lucía, que tienen más de diez años de experiencia como maestras. Por tanto, puntuar mejor o peor en un criterio, en concreto (es decir orientar su práctica de acuerdo a este criterio), no parece que tenga que ver con la experiencia.

También se puede observar en los resultados mostrados en la Tabla 6, que en el caso del criterio ecológico, más de la mitad de los maestros/as participantes consiguen puntuaciones mayores o iguales a 1.5 (es decir, que sus secuencias didácticas sí que tienen en cuenta criterios ecológicos tales como la adaptación del currículum, las conexiones inter e intradisciplinares etc.). Esta tendencia se mantiene también cuando revisamos este dato controlando por la experiencia docente de cada sujeto participante en el estudio. En cambio, en el caso de las maestras que menos años han estado trabajando como tales (o que todavía nunca trabajaron como maestras), las puntuaciones revelan que el criterio ecológico está poco presente en sus secuencias didácticas. Esto sugiere que la falta de experiencia podría explicar que los maestros/as tiendan a diseñar lecciones basados en criterios propios, que pueden (o no) estar alineados con las directrices curriculares. Pero, cuando ya se tiene una cierta experiencia como maestro/a, es más habitual que se tenga en cuenta el currículum y la interdisciplinariedad en el diseño de secuencias didácticas y también la adaptación al entorno sociolaboral de sus alumnos.

En el caso del criterio epistémico, no se observa ninguna tendencia relevante. En todos los casos, salvo en Rubén y Lina, las puntuaciones están alrededor del 2, lo que significa que todos los maestros y maestras participantes en el estudio sí que han tenido en cuenta este criterio para orientar sus diseños didácticos. Se trata de un dato interesante, por cuanto que la investigación reciente sobre la formación matemática de los maestros y maestras en Panamá sugiere que existe un claro déficit en este ámbito (MORALES MAURE, 2019). Este dato sugiere que el paso por el Diplomado sirve para que los maestros/as que participan en él mejoren su formación matemática, y eso se refleja en las lecciones (secuencias didácticas) que diseñan e implementan. Este resultado avala, por tanto, el trabajo que se está haciendo desde el Diplomado, por la mejora de la enseñanza de las matemáticas en Panamá.

$\mathrm{Al}$ analizar el criterio cognitivo volvemos a encontrar un aspecto interesante: en este caso, los resultados sugieren que prácticamente todos los maestros y maestras participantes en el estudio han tenido en cuenta aspectos cognitivos del aprendizaje de las matemáticas de sus alumnos. Únicamente en tres casos (Miriam, Oris y Mercedes), las puntuaciones bajan del 2. Parece que cuantos más años de experiencia, mejores puntuaciones se obtienen, cosa que sugiere que sus lecciones son más idoneas desde el punto de vista de tener en cuenta los aspectos 
cognitivos (es decir, aparecen con mayor frecuencia componentes tales como tener en cuenta los conocimientos previos, la adaptación curricular a las diferencias individuales, el aprendizaje o el planteamiento de tareas con alta demanda cognitiva).

En cambio, en el caso de Oris y de Mercedes, con nula experiencia como maestras, todavía, la puntuación en este criterio de sus respectivas secuencias didácticas se sitúa por debajo del 2, lo que sugiere que les falta, todavía, formación y/o pericia para introducir elementos de complejidad matemática en sus propuestas. El análisis de sus respectivas secuencias didácticas ilustra planteamientos vagos, ambiguos o poco concretos.

Los resultados obtenidos en el caso del criterio afectivo son completamente diferentes. En este caso, casi en todas las secuencias didácticas hemos encontrado la presencia de elementos que sugieren una idoneidad afectiva total. Parece ser que todos los maestros y maestras que han participado en el estudio han puesto especial esfuerzo en cuidar que las actividades planteadas fuesen atractivas, que respondiesen (desde su punto de vista) a las necesidades o intereses de sus estudiantes, y que evitasen situaciones de fustración, o sentimiento negativo hacia las matemáticas. Esto sugiere que los maestros y las maestras que han participado son, de alguna manera, muy permeables a esa parte del discurso social que existe en torno a las matemáticas que las presenta como algo negativo, que produce miedo, aburrimiento, desapego etc.

Para evitarlo, los maestros y las maestras han procurado que sus lecciones se pudiesen definir como unas matemáticas falibilistas (en términos de Ernest), o unas matemáticas sociales, culturales o socioculturalmente situadas (de acuerdo con otros puntos de vista también conocidos en la didáctica de las matemáticas, como son las posiciones de LERMAN, 2000; BISHOP, 1991; NUNES; CARRAHER; SCHLIEMANN, 1993; D'AMBROSIO, 1985; YAECKEL; COBB,1996; CLARKE; KEITEL; SHIMIZU, 2006). Los años de experiencia no hacen cambiar el sentido de la relación encontrada por lo que respecta a este criterio.

En el caso del criterio interaccional, no parece, tampoco, que se pueda establecer algún tipo de patrón o regularidad. En general, todos los sujetos participantes en el estudio muestran que tienen en cuenta este criterio para orientar sus respectivas secuencias didácticas. De hecho, los planteamientos de cómo establecer el tipo de interacción maestro-alumno, y alumnoalumno, forman parte de la formación que han recibido (i.e. a través del tipo de tareas que pueden plantear a sus estudiantes); por tanto, no es de extrañar que este criterio aparezca claramente en sus planteamientos, como revela que casi todos los maestros/as obtengan más de 2 puntos o 2.25 puntos en él.

Únicamente sorprendren los casos de Miriam y Lina, maestras con menos experiencia, 
cuyas puntuaciones están ligeramente por debajo de 2. Atribuimos este hecho, quizás, a su falta de experiencia, que se refleja también en la limitación de ponerse en situación de entablar diálogo con sus estudiantes, cuando implementan sus respectivas lecciones. Al contrario, se las ve sobre todo adoptando un papel más tradicional de explicación unidireccional (desde el pizarrón hacia sus estudiantes). Quizás, sea debido a la falta de práctica, aunque se tendría que comprobar en estudios futuros.

Finalmente, por lo que respecta al criterio mediacional relativo a los recursos y materiales usados en clase, en todos los casos (menos uno) los maestros/as obtienen la máxima puntuación. Ello es debido, desde nuestro punto de vista, a que el Diplomado pone mucho énfasis en diversificar los materiales manipulativos como recursos de enseñanza y aprendizaje de las matemáticas, de acuerdo con su orientación hacia la innovación educativa. Por eso, el obtener estas puntuaciones es un indicador también del éxito del Diplomado, desde el punto de vista de formar en el uso de materiales, que permite una docencia más allá de la clase teórica o teórico/demostrativa en la pizarra, con métodos tradicionales (razonamiento deductivo, demostraciones, ejemplos y repetición de los mismos - drill and practice -).

\section{Consideraciones Finales}

El análisis presentado en este artículo sugiere que la experiencia docente que tienen los maestros y las maestras de matemáticas que han participado en este estudio puede explicar por qué obtienen unos índices-CID más elevados, lo que quiere decir que sus secuencias didácticas (sus lecciones) son más idóneas que las que diseñan e implementan los/as maestros/as con menos experiencia. Una posible explicación de este fenómeno, que convendría investigar más en futuros estudios, es que la experiencia lo que hace es que los maestros y las maestras vayan consolidando prácticas y estrategias que les han funcionado en el pasado, desechando aquéllas que no les sirvieron para sus propósitos didácticos.

Los criterios CID nacen de un amplio consenso con la comunidad internacional de maestros y maestras (e investigadores/as) que han plasmado en los criterios y sus componentes todas aquellas prácticas (y sus indicadores) sobre las que hay acuerdo de que funcionan (GODINO; BATANERO; FONT, 2020). La idoneidad se concreta como consensos a los que se llega sobre aquello que funciona en el aula de matemáticas. Los resultados que hemos obtenido en este estudio sugieren que las maestras con más experiencia, son las que más fácilmente realizan las prácticas que se consideran más idóneas, y eso lo reflejan en sus secuencias didácticas. 
Por otro lado, otro aspecto relevante que destaca de este estudio exploratorio es la importancia de la participación en programas de desarrollo profesional docente, como el Diplomado. Los resultados (sobre todo los referentes a los criterios epistémico, cognitivo, mediacional e interaccional), sugieren que las maestras y los maestros de nuestro estudio han alcanzado puntuaciones elevadas en cada una de ellos . Los datos previos existentes en Panamá (MORALES MAURE, 2019) sugieren que los docentes panameños, en general, tienen déficits en su formación (matemática y como docentes). Pero cuando se observa el resultado de su paso por el Diplomado (a través del análisis del trabajo final, que es la secuencia didáctica implementada), se observa que en criterios clave tan importantes para orientar su práctica como el epistémico (relativo al conocimiento de las matemáticas), el cognitivo (sobre el conocimiento de cómo los estudiantes aprenden), el mediacional (sobre el uso de materiales y recursos didácticos para la enseñanza de las matemáticas), y el interaccional (sobre el impacto crucial de las interacciones en el aprendizaje, tal y como muestra investigaciones previas como el de DÍEZ-PALOMAR; CABRÉ, 2015), las maestras y los maestros han obtenido puntuaciones elevadas (superiores en muchos casos a 2). Esto indica que han logrado aprovechar las enseñanzas del Diplomado. Y, por otro lado, que los CID han servido para dar cuenta de ese aprovechamiento, como herramienta de evaluación didáctica.

Otro aspecto que ha revelado el uso de los CID ha sido el papel importante que juega el criterio de idoneidad afectiva en el diseño e implementación de las secuencias didácticas de matemáticas. Quizás, sea debido a que los maestros y las maestras que han participado en el estudio muestran una sensibilidad hacia el discurso de las matemáticas como una asignatura difícil, que otros estudios previos ya han puesto de relieve (TUNCER; YILMAZ, 2020). Los datos indican que todas las maestras y maestros del estudio diseñaron secuencias didácticas que pretendían gustar, implicar, resultar atractivas, para sus estudiantes.

En algunos casos eso se ha combinado con una presentación rigurosa de las matemáticas, y teniendo en cuenta aspectos de su didáctica. En otros casos (sobre todo en las maestras con menos experiencia), o bien ha fallado la parte de la matemática (criterio epistémico), o bien han diseñado secuencias didácticas, pero sin tener en cuenta aspectos de la enseñanza y aprendizaje de la matemática (criterio cognitivo). Los resultados indican que la menor experiencia hace que los maestros y las maestras planteen unas lecciones basadas en sus criterios propios, no en las evidencias científicas (en el conocimiento didáctico de la materia), y eso puede llevar a que traten de llevar a cabo prácticas que didácticamente hablando no son las más idóneas.

Finalmente, un último aspecto a destacar del análisis de los resultados es el impacto 
claro que ha tenido el Diplomado en la introducción de elementos de innovación didáctica en las secuencias diseñadas. Todos los maestros/as que han participado en el estudio han incluido elementos del criterio mediacional, que se refiere a los recursos usados en el aula durante la lección. En todos los casos, menos en el de Lina, el índice de este criterio ha sido de 3 (la máxima puntuación). Los CID nos permiten observar que el paso por el Diplomado ha tenido, también, el efecto que los maestros y las maestras han interiorizado el uso de recursos (sobre todo) y formas de interacción innovadoras.

Todo esto nos permite afirmar que los CID, tal y como los hemos utilizado aquí (a través de los índices desarrollados), parece que sí, que sirven para evaluar algunos de los resultados de un programa como el Diplomado en la formación profesional de los docentes de matemáticas en Panamá. Habrá que ver, en futuros estudios, si esta afirmación se puede generalizar más allá de los diez individuos que han participado en este estudio, y la podemos extender no solo al resto de docentes panameños, sino a docentes en otros países, contextos, culturas etc.

\section{Agradecimientos}

Esta investigación cuenta con el financiamiento de la Vicerrectoría de Investigación y Postgrado de la Universidad de Panamá (UP) con los fondos ganados en el 2019 código VIP01-04-01-2019-11, el investigador principal es becario por Secretaría Nacional de Ciencia, Tecnología e Innovación (SENACYT) del doctorado en la Universitat de Barcelona y todos los autores son miembros del Grupo de Investigación en Educación Matemática - GIEM21 de la UP.

Además, este trabajo ha sido elaborado con apoyo del proyecto de intervención: Convenio de Colaboración Educativa N. ${ }^{\circ}$ 40-2019 entre la SENACYT y la Universidad de Panamá y con Aval del Ministerio de Educación. Bajo el financiamiento para el programa de Innovación para la Inclusión Social y la Productividad de la República de Panamá.

También queremos agradecer la colaboración del proyecto de investigación PGC2018098603-B-I00, financiado por el Ministerio de Ciencia, Innovación y Universidades, del Gobierno de España.

Asimismo, también queremos reconocer el apoyo del Dr. Jaime Javier Gutiérrez Vicerrector de Investigación y Postgrado, Mgtr. Denis Chávez - Vicerrector de Extensión, la Dra. María Heller (Directora de Aprendizaje-SENACYT) y María Trinidad Jiménez (Coordinadora del BID-SENACYT).

Con la colaboración del Lic. Emilce Mejía (Coordinadora de Educación-ESRI Panamá). 


\section{Referencias}

ALSINA, A.; DOMINGO, M. Idoneidad didáctica de un protocolo sociocultural de enseñanza y aprendizaje de las matemáticas. Revista Latinoamericana de Investigación en Matemática Educativa, Ciudad de México, v. 13, n. 1, p. 7-32, mar. 2010.

BISHOP, A. Mathematical enculturation: A cultural perspective on mathematics education. Dordrecht: Springer, 1991.

BLANCO, T. F.; GODINO, J. D.; DIEGO-MANTECÓN, J. Análisis epistémico y cognitivo de una tarea de visualización en el espacio bidimensional. REDIMAT, Barcelona v. 7, n. 3, p. 251-279, oct. 2018.

BREDA, A. Características del análisis didáctico realizado por profesores para justificar la mejora en la enseñanza de las matemáticas. Bolema, Rio Claro, v. 34, n. 66, p. 69-88, apr. 2020.

BREDA, A.; FONT, V.; PINO-FAN, L. Criterios valorativos y normativos en la Didáctica de las Matemáticas: el caso del constructo idoneidad didáctica. Bolema, Rio Claro, v. 32, n. 60, p. 255-278, apr. 2018.

BREDA, A.; PINO-FAN, L.; FONT, V. Meta didactic-mathematical knowledge of teachers: criteria for the reflections and assessment on teaching practice. Eurasia Journal of Mathematics, Science and Technology Education, Eastbourne, v. 13, n, 6, p. 1893-1918, jun. 2017.

CAMPUS VIRTUAL - UNIVERSIDAD DE PANAMÁ. Diplomado en Estrategias para la Enseñanza de la Matemática. 2019. Disponible en: https://upanama.e-ducativa.com/ index.cgi?id_curso=3348. Acceso: 20 feb. 2020.

CARPENTER, T. P.; FENNEMA, E.; PETERSON, P. L.; CAREY, D. A. Teachers' pedagogical content knowledge of students' problem solving in elementary arithmetic. Journal for Research in Mathematics Education, Reston, v. 19, n. 5, p. 385-401, nov. 1988.

CLARKE, D.; KEITEL, C.; SHIMIZU, Y. Mathematics classrooms in twelve countries: The insider's perspective. Rotterdam and Taipei: Sense Publishers, 2006.

CRUZ, A.; GEA, M.; GIACOMONE B. Criterios de idoneidad epistémica para el estudio de la geometría espacial en educación primaria. En: CONTRERAS, N. J. M.; ARTEAGA, P.; CAÑADAS, G. R.; GEA, M. M.; GIACOMONE, B.; LÓPEZ-MARTÍN, M. M. (Eds.) Actas del Segundo Congreso International Virtual sobre el Enfoque Ontosemiótico del Conocimiento y la Instrucción Matemáticos. Granada: Civeos, 2017, p. 1-10.

D'AMBROSIO, U. Ethnomathematics and its place in the history and pedagogy of mathematics. For the learning of mathematics, New York, v. 5, n.1, p. 44-48, feb. 1985.

PONTE, J. P. Investigating mathematics and learning to teach mathematics. In: LIN, F. L.; TCOONEY, T. J. (Eds.). Making sense of mathematics teacher education. Dordrecht: Springer, 2001. p. 53-72.

DÍEZ-PALOMAR, J.; CABRÉ, J. Using dialogic talk to teach mathematics: The case of interactive groups. ZDM Mathematics Education, Berlín, 7, p. 1299-1312, nov. 2015.

DIEZ-PALOMAR, J.; VANEGAS, Y.; GIMÉNEZ, J.; FONT, V. Análisis del criterio de idoneidad ecológica en la formación del profesorado de matemáticas. 2014. Disponíble en: http://amieedu.org/actascimie14/?cat=3 Acessado el: 1 Jul. 2021. 
ESQUÉ, D.; BREDA, A. Valoración y rediseño de una unidad sobre proporcionalidad utilizando la herramienta Idoneidad Didáctica. Uniciencia, Heredia, v. 35, n. 1, p. 38-54, jan./jun. 2021.

ESRI-PANAMÁ. Educación 507. 2019. Disponible en:

https://educacion507.maps.arcgis.com/home/webmap/viewer.html?webmap=558d426addce43c69e1 a5 ba9870d200f. Acceso: 5 jun. 2020.

EVEN, R.; BALL, D. (Eds.). The professional education and development of teachers of mathematics. New York: Springer, 2009.

FONT, V. Criterios valorativos y normativos en la didáctica de una disciplina científica. Cuadernos de Investigación y Formación en Educación Matemática, San José, v. 14, n. 18, p. 151-161, dec. 2019.

FONT, V.; BREDA, A.; SECKEL, M.J.; PINO-FAN, L. Análisis de las reflexiones y valoraciones de una futura profesora de matemáticas sobre la práctica docente. Revista de Ciencia y Tecnología, San José C. R., v. 34, n. 2, p. 62-75, 2018.

FONT, V; ADÁN, M.; FERRERES, S. Valoración de la idoneidad de las Matemáticas enseñadas. En: SCOTT, P.; RUÍZ, A. (Eds.). Educación Matemática en las Américas: 2015. Volumen 2: Formación Inicial para Secundaria. República Dominicana: Comité Interamericano de Educación Matemática, 2015. p. 338-345.

GIACOMONE, B.; GODINO, J. D.; BELTRÁN-PELLICER, P. Developing the prospective mathematics teachers' didactical suitability analysis competence. Educação e Pesquisa, São Paulo, v. 44, e172011, p. 1-21, 2018.

GODINO, J. D. Indicadores de la idoneidad didáctica de procesos de enseñanza y aprendizaje de las matemáticas. In: CIAEM-IACME, 13, 2011, Recife, Brasil. Anais... Recife: Universidade Federal de Pernambuco, 2011. p. 1-20

GODINO, J. D. Indicadores de la idoneidad didáctica de procesos de enseñanza y aprendizaje de las matemáticas. Cuadernos de Investigación y Formación en Educación Matemática, San José C.R., v. 8, n. 11, p. 111-132, dic. 2013.

GODINO, J. D.; BATANERO, C.; FONT, V. Un enfoque ontosemiótico del conocimiento y la instrucción matemática. 2007. Disponible en: http://www.ugr.es/ jgodino/funciones semioticas/sintesis_eos_10marzo08.pdf. Acceso: 03 mar. 2020

GODINO, J. D.; BATANERO, C.; FONT, V. The onto-semiotic approach: implications for the prescriptive character of didactics. For the Learning of Mathematics, Edmonton, v. 39, n.1, p. 3843, 2019.

GODINO, J. D.; BATANERO, C.; FONT, V. El Enfoque ontosemiótico: implicaciones sobre el carácter prescriptivo de la didáctica. Revista Chilena de Educación Matemática, Valparaíso, v. 12, n. 2, p. 47-59, abr. 2020. DOI: https://doi.org/10.46219/rechiem.v12i2.25.

GODINO, J. D.; GIACOMONE, B.; BATANERO, C.; FONT, V. Enfoque ontosemiótico de los conocimientos y competencias del profesor de matemáticas. Bolema, Rio Claro, v. 31, n. 57, p. 90113, april 2017.

HILL, H. C.; BALL, D. L.; SCHILLING, S. G. Unpacking pedagogical content knowledge: Conceptualizing and measuring teachers' topic-specific knowledge of students. Journal for Research in Mathematics Education, Reston, v. 39, n. 4, p. 372-400, jul. 2008. 
KUNTER, M.; KLUSMANN, U.; BAUMERT, J.; RICHTER, D.; VOSS, T.; HACHFELD, A. Professional competence of teachers: Effects on instructional quality and student development. Journal of Educational Psychology, Washington D.C., v. 105, n. 3, p. 805-820, aug. 2013.

LERMAN, S. Accounting for accounts of learning mathematics: Reading the ZPD in videos and transcripts. In: CLARKE, D. (Ed.). Perspectives on practice and meaning in mathematics and science classrooms. Springer: Dordrecht, 2001. p. 53-74.

LERMAN, S. The social turn in mathematics education research. In: BOALER, J. (Ed.). Multiple Perspectives on Mathematics Teaching and Learning. London: Ablex Publishing, 2000. p. 19-44.

LLINARES, S. Construcción de conocimiento y desarrollo de una Mirada profesional para la práctica de enseñar matemáticas en entornos en línea. Avances de Investigación en Educación Matemática, Granada, v. 1, n. 2, p. 53-70, nov. 2012.

LLINARES, S. Professional noticing: A component of the mathematics teacher's professional practice. Sisyphus-Journal of Education, Lisboa, v. 1, n. 3, p. 76-93, 2013.

LÓPEZ, J. Idoneidad Mediacional de Futuros Maestros de Matemáticas. 2016. Trabajo de Conclusión (Grado de Licenciatura) - Universidad de Antioquia, Facultad de Educación, Medellín, 2016.

MASON, J. Noticing: Roots and branches. In: SHERIN, M. G.; JACOBS, V. R.; PHILLIPP, R. A. (Eds.) Mathematics teacher noticing: Seeing through teachers' eyes. New York: Routledge, 2011. p. 35-50.

MORALES-LÓPEZ, Y.; FONT, V. Valoración realizada por una profesora de la idoneidad de su clase de matemáticas. Educação e Pesquisa, São Paulo, v. 45, e189468, p. 1-10, 2019.

MORALES MAURE, L.; GARCÍA VÁSQUEZ, E.; DURÁN GONZÁLEZ, R. Intervención formativa para el aprendizaje de las matemáticas: una aproximación desde un diplomado. Conrado, Revista pedagógica de la Universidad de Cienfuegos, Cienfuegos, v. 15, n. 69, p. 7-18, 2019.

MORALES MAURE, L. M. Competencia de análisis e intervención didáctica del docente de primaria en Panamá. 2019. 309 f. Tesis (Doctorado en Didáctica de las Matemáticas) - Facultat de Formació del Professorat, Universitat de Barcelona, Barcelona, 2019.

NUNES, T.; CARRAHER, D.; SCHLIEMANN, A. D. Street mathematics and school mathematics. Cambridge: Cambridge University Press, 1993.

OH, H. S.; SEO, D. I.; KIM, J. S.; YOO, S. O.; SEONG, H. C. Assessment and evaluation of national human resource development system competitiveness in emerging countries. Asia Pacific Education Review, Seoul, v. 16, n. 3, p. 477-490, 2015.

PINO-FAN, L.; FONT, V.; BREDA, A. Mathematics teachers' knowledge and competences model based on the onto-semiotic approach. In: CONFERENCE OF THE INTERNATIONAL GROUP FOR THE PSYCHOLOGY OF MATHEMATICS EDUCATION, 41, 2017, Singapore. Proceedings [...] Singapore: PME, 2017, v. 4, p. 33-40.

PIRIE, S. E. B. “Where do We Go from Here?". In: TEPPO, A. R. (Ed.). Qualitative Research Methods in Mathematics Education. Reston: National Council of Teachers of Mathematics, 1998. p. $156-163$. 
PLANAS, N. Modelo de análisis de videos para el estudio de procesos de construcción de conocimiento matemático. Educación Matemática, Ciudad de Mexico, v. 18, n. 1, p. 37-72, 2006.

ROWLAND, T.; TURNER, F.; THWAITES, A.; HUCKSTEP, P. Developing primary mathematics teaching: Reflecting on practice with the Knowledge Quartet. Londres: SAGE, 2009.

SCHÖN, D. A. The Reflective Practitioner.: How Professionals Think in Action. Avebury: Aldershot Hants, 1983.

SECKEL, M. J. Competencia en análisis didáctico en la formación inicial de profesores de educación general básica con mención en matemática. 2016. Tesis (Doctorado en Didáctica de las Matemáticas) - Facultat de Formació del Professorat, Universitat de Barcelona, Barcelona, 2015.

SECKEL, M. J.; BREDA, A.; SÁNCHEZ, A.; FONT, V. Criterios asumidos por profesores cuando argumentan sobre la creatividad matemática, Educação e Pesquisa, São Paulo, v. 45, p.1-18, jan. 2019.

SHULMAN, L. S. Those who understand: Knowledge growth in teaching. Educational Rresearcher, v. 15, n. 2, p. 4-14, feb. 1986.

SILVERMAN, J.; THOMPSON, P. Toward a framework for the development of mathematical knowledge for teaching. Journal of mathematics teacher education, Dordrecht, v. 11, n. 6, p. 499511, nov. 2008.

TUNCER, M.; YILMAZ, Ö. Relations attitude towards mathematics lesson: anxiety and academic success. REDIMAT, Barcelona, v. 9, n. 2, p. 173-195, 2020.

ÜNVER, S. K.; ÖZGÜR, Z., GÜZEL, E. B. Investigating Preservice Mathematics Teachers' Pedagogical Content Knowledge through Microteaching. REDIMAT, Barcelona, v. 9, n. 1, p. 62-87, feb. 2020.

YAECKEL, E.; COBB, P. Sociomathematical norms, argumentation, and autonomy in mathematics. Journal for Research in Mathematics Education, Reston, v. 27, n. 4, p. 458-477, jul. 1996.

YOUNG, J. R.; YOUNG, J.; HAMILTON, C.; PRATT, S. S. Evaluating the effects of professional development on urban mathematics teachers TPACK using confidence intervals. REDIMAT, Barcelona, v. 8, n. 3, p. 312-338, oct. 2019. 